Case Report

\title{
Management of Agitation, Delirium, and Catatonia in Intubated COVID- 19 Patients: A Case Series \& Rationale for Valproate Sodium Use During Extubation
}

Ashley Schumaker ${ }^{1}$, Richard Gomez 2, Sofia Rojasova ${ }^{2}$, Cassidy Muir 2, Katie Bukiri ${ }^{1}$, Clinton Korneffel ${ }^{1}$, Aparna Sharma ${ }^{1}$, Murali Rao ${ }^{1}$, Edwin Meresh ${ }^{1,}{ }^{*}$

1. Loyola University Medical Center, 2160 S. First Ave, Maywood, IL 60153, Illinois, USA; E-Mails: Ashley.Schumaker001@luhs.org; Katiebukiri@gmail.com; Clinton.E.Korneffel@luhs.org; apsharma@lumc.edu; Mrao1@lumc.edu; emeresh@lumc.edu

2. Loyola University Stritch School of Medicine, 2160 S. First Ave, Maywood, IL 60153, Illinois, USA; E-Mails: Rgomez8@luc.edu; srojasova@luc.edu; cmuir@luc.edu

* Correspondence: Edwin Meresh; E-Mail: emeresh@lumc.edu

Academic Editor: Bart Ellenbroek

OBM Neurobiology

2021, volume 5 , issue 2

doi:10.21926/obm.neurobiol.2102099
Received: February 25, 2021

Accepted: May 14, 2021

Published: May 25, 2021

\section{Abstract}

The complete spectrum of neuropsychiatric effects of SARS-CoV-2 acute respiratory distress syndrome has yet to be fully appreciated, particularly in intubated patients. Manifestations including delirium and catatonia need to be considered in intubated COVID-19 patients. Medications known to exhibit neuroprotective effects, like valproate, can assist in agitation related to sedative withdrawal during extubation. This case series reports on the management of agitation, delirium, and catatonia in COVID-19 patients during and after extubation efforts. We present three cases in which Psychiatry was consulted for agitation in intubated COVID19 patients. These patients were managed for severe agitation during weaning from extubation as well as for subsequent psychiatric challenges, including catatonia. Patient 1: 26year-old female with bipolar I with psychotic features who was admitted for acute hypoxic respiratory failure from COVID-19 pneumonia. After an emergency C-section at 31 weeks' gestation, she was intubated for 9 days and started on valproate $250 \mathrm{mg}$ BID for agitation. She

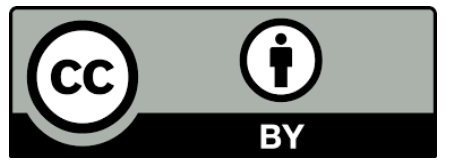

(C) 2021 by the author. This is an open access article distributed under the conditions of the Creative Commons by Attribution License, which permits unrestricted use, distribution, and reproduction in any medium or format, provided the original work is correctly cited. 
was extubated successfully and discharged home. Patient 2: 42-year-old female with bipolar I and PTSD who was intubated following COVID-19-related acute hypoxic respiratory failure. She received valproate $250 \mathrm{mg}$ BID and was extubated successfully. She became catatonic when home quetiapine was resumed and recovered following quetiapine discontinuation and lorazepam. She was discharged on valproic acid and alprazolam. Patient 3: 23-year-old female with bipolar I with psychotic features who was admitted for COVID-19 acute hypoxic respiratory failure and intubated. She received valproate $250 \mathrm{mg}$ BID and was extubated successfully. She became catatonic when risperidone was re-initiated, but recovered following risperidone discontinuation and addition of lorazepam, gabapentin, and duloxetine. Quetiapine was added before discharge. Valproate was effective for managing delirium and agitation during extubation, as evidenced by normalizing Richmond Agitation and Sedation Scale scores. Additionally, valproate aided in managing catatonia post-extubation. This case series reports on the management of agitation in COVID-19 patients during extubation efforts with valproate sodium, due to its ability to manage delirium and catatonia. Valproate is known to exhibit neuroprotective effects, which possibly explains successful management of agitation during the extubation process.

\section{Keywords}

COVID-19; catatonia; delirium; valproic acid; extubation; neuroinflammation

\section{Background}

As the COVID-19 pandemic continues to cause significant global mortality and morbidity, the complete spectrum of neuropsychiatric effects of SARS-CoV-2 acute respiratory distress syndrome has yet to be fully appreciated. Neuropsychiatric complications have been reported and neuroinflammation has been mechanistically implicated in COVID-19 [1, 2]. Medications known to exhibit neuroprotective effects, such as valproate, could contribute to successful management [3]. This case series reports on the management of concurrent delirium and catatonia in COVID-19 patients before, during, and after extubation. In the discussion, we argue that valproate sodium showed success in these settings and was an appropriate choice throughout care due to its ability to manage both delirium and catatonia. The first documented case of catatonia and co-ocurring delirium in a COVID-19 patient also saw a favorable response to repeated doses of lorazepam [4]. However, benzodiazepines are known to worsen delirium so this may not be a sufficient therapy to treat both processes [5]. As these patients were intubated secondary to COVID-19 pneumonia, the progression of psychiatric disease was at times difficult to evaluate. If the patients were having catatonia (alone or concurrently with delirium), antipsychotics may have worsened their status. On the other hand, valproic acid did not carry the risk of worsening the catatonia.

Moreover, dexamethasone and sedatives were used in these cases due to complex medical and psychiatric histories, and hospital courses complicated by COVID-19. Systemic dexamethasone has known neuropsychiatric side effects, which most commonly present as hypomania or euphoria, but also include mood, sleep, and behavioral disturbances such as agitation, or psychosis and delirium in severe cases $[5,6]$. A meta-analysis found that mild-moderate dexamethasone reactions can 
occur in up to $28 \%$ of patients, while severe reactions can occur in $6 \%$ of patients [6]. A myriad of other medications--including, but not limited to, sedatives like benzodiazepines, opioids, anesthetics, antihistamines, and anticholinergics--can also induce psychotic symptoms [5].

Furthermore, it has been proposed that neuroinflammation from COVID-19 leads to increased risk of delirium and catatonia [7]. There have been reports of COVID-19 patients who develop catatonia, as well as suggestions that catatonia remains underdiagnosed in COVID-19 patients due to medical complexity and limited psychiatric and physical exams $[8,9,10]$. As exemplified by the patients in this case series and explored in the discussion, there is possible increased risk of catatonia in COVID-19 patients. Additionally, a study of critically ill patients has shown that an increased number of signs of catatonia in a patient were associated with a greater likelihood of concurrent delirium [11]. The pathophysiology of COVID-19 and its consequences on the central nervous system (CNS) may provide an explanation for increased risk of the aforementioned neuropsychiatric findings. In patients with COVID-19, there is an infiltration of monocytes and neutrophils into the CNS that leads to disruption and increased permeability of the blood-brain barrier (BBB) [7]. This inflammatory response may lead to poorer neurological outcomes, including onset of delirium or agitation. One theory is that increased oxidative stress caused by COVID-19 leads to infection and immune responses such as endothelial transfer, cytokine storm, and BBB breakdown. In addition to dysregulation, stress hormones of the hypothalamus can cause encephalopathy, manifesting as agitation, sleep-wake cycle disturbances, abulia, and motor disorders [7]. Both the robust immune response and the neuropsychiatric side effects of corticosteroids (used in Case 3) and sedatives (used in Case 1, 2, and 3) increased the likelihood that the patients in this case series could simultaneously experience catatonia and delirium during the course of their illness [7]. In addition to the neuroinflammatory hypothesis, there are several other possible mechanisms that could have led or contributed to delirium and/or catatonia in COVID-19 patients, including environmental factors (e.g., ICU setting), iatrogenic contributors (e.g, sedating medications), and social isolation status (e.g., prohibited family visitation). 
2. Case Studies (Table 1)

Table 1 Overview of three cases with highlighted similarities. Details are in the body of report.

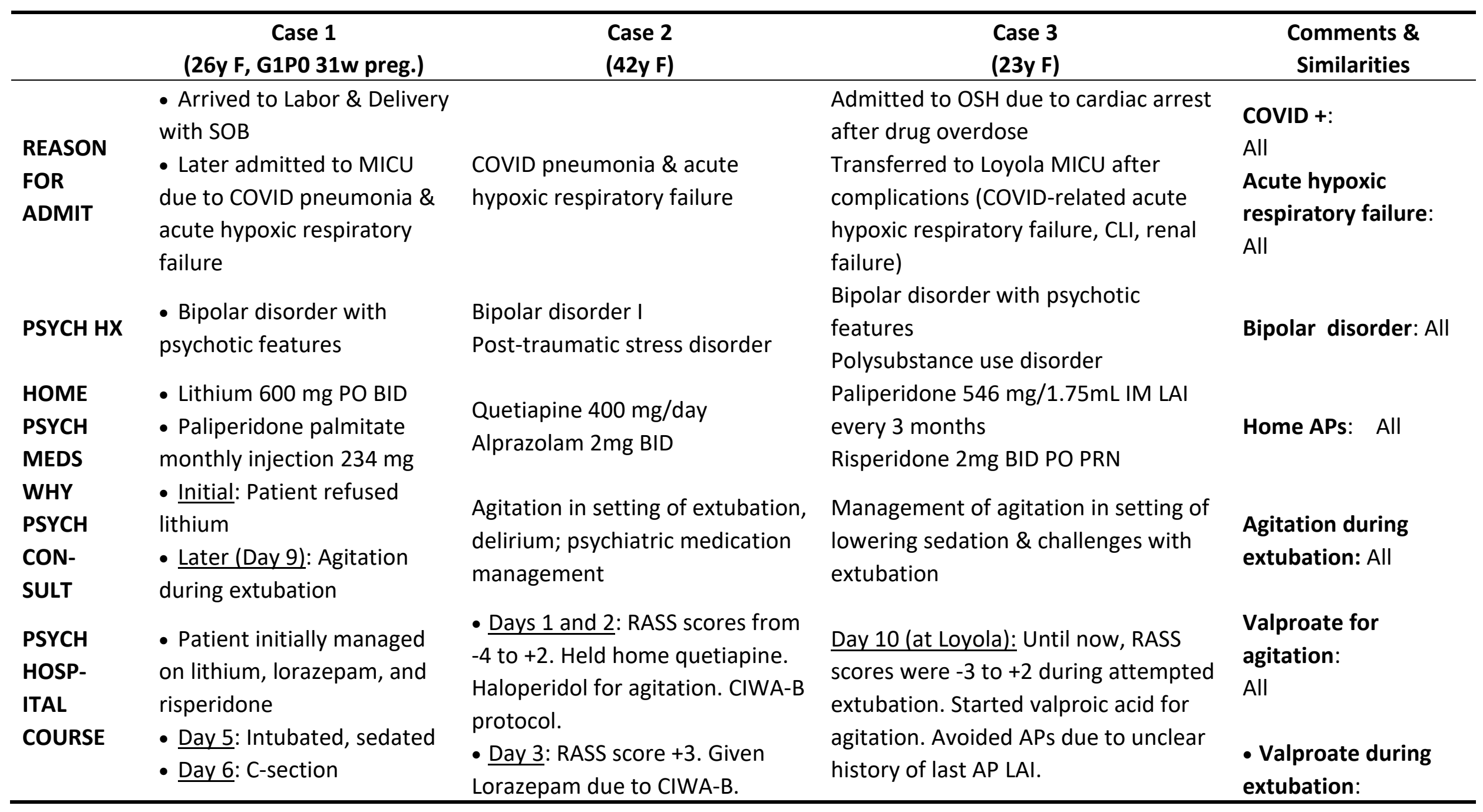


- Day 6 to 9: RASS scores from -3 to -1 while on lithium, lorazepam, and risperidone. Haloperidol PRN added for agitation.

- Day 10 to 13: RASS scores from -1 to +2 . Discontinued haloperidol and lorazepam (respiratory concerns). Started valproate sodium for agitation

- Day 14: Extubated after 2 valproate doses. RASS scores from -1 to 0. Discontinued risperidone.

- Day 15: Discontinued valproate after 2 final doses. RASS score stabilized at 0 . Discharged home on lithium and paliperidone palmitate administration.
Haloperidol IV and lorazepam PRN for agitation.

- Day 4: Haloperidol stopped due to prolonged QTC. Started valproate sodium.

- Days 5-8: Continued valproate as RASS scores from +2 to -1 , stabilized to 0 .

- Day 6: Extubated after 3 valproate sodium doses. Switched to valproic acid until Day 10, then stopped.

- Day 11: Quetiapine restarted. Subsequent fever (100.4 F), tachycardia (140), drooling, staring, shuffled gait; discontinued quetiapine. Restarted valproic acid and started lorazepam for 5 days. - Day 17: Catatonia resolved. Valproic acid continued. Quetiapine discontinued. Lorazepam weaned off, patient discharged with home alprazolam and Depakote.
Day 11: Increased valproic acid. Patient self extubated. RASS scores from 0 to +1 . Started risperidone for bipolar disorder.

Day 12-15: Continued valproic acid and risperidone.

Day 16: Discontinued valproic acid due to surgical site bleeding and labs (low $\mathrm{Hgb})$.

Day 17: Outpatient psych confirmed home paliperidone LAI and risperidone. Held paliperidone LAI because of long-acting effects and potential recovery time. Held risperidone due to signs of possible NMS.

Day 18: Pt had cogwheel rigidity and catatonia. Started trial of lorazepam for catatonia.

Day 19 to 24: Concern for febrile catatonia. Increased lorazepam.

Long-term Course: Patient being weaned off diazepam. Gabapentin and duloxetine added. Added quetiapine before discharge.

$\mathrm{SOB}=$ shortness of breath; $\mathrm{MICU}=$ medical intensive care unit; OSH = outside hospital; $\mathrm{CLI}=$ chronic limb ischemia; $\mathrm{LAI}=$ long acting injectable; $\mathrm{AP}=$ antipsychotic; CIWA-B = Clinical Institute Withdrawal Assessment for Benzodiazepines; NMS = neuroleptic malignant syndrome 


\subsection{Case 1}

Case 1 concerns a 26-year-old pregnant female at 31 weeks gestation, with a history of bipolar disorder with psychotic features, treated with lithium $600 \mathrm{mg}$ PO twice daily and a paliperidone long-acting injectable (LAI) 234 mg monthly. The patient was previously on Valproic acid for bipolar disorder, but this medication was discontinued, when she became pregnant, due to concerns for teratogenicity. She presented to the emergency department with cough and shortness of breath and was subsequently admitted to the Medical Intensive Care Unit for continued management of COVID pneumonia and acute hypoxic respiratory failure. She was then admitted to the Labor and Delivery unit for continued fetal monitoring.

Psychiatry consult services were utilized when the patient refused to take her lithium regimen. The psychiatry team provided psychoeducation, and after expressing understanding, she resumed her daily lithium for bipolar disorder, risperidone for psychosis, and lorazepam and haloperidol PRN for agitation. On day 5, she was initially placed on a nonrebreather, due to oxygen desaturation, but because of a lack of response, she was subsequently intubated. The patient then delivered her infant on day 6 via cesarean delivery. RAAS scores ranged from -3 to -1 days 6 through 9. On day 9, she began displaying signs of agitation with efforts at extubation, as evidenced by Richmond Agitation and Sedation Scale (RASS) scores ranging from -1 to +2 . Psychiatry was then consulted for managing agitation in the setting of attempted extubation. Up to this point in her hospitalization, the patient received one dose of IV lorazepam $2 \mathrm{mg}$ PRN and multiple PO doses, totaling $34 \mathrm{mg}$; however, her lack of response compelled the team to switch therapy to Valproate to help manage agitation. Therefore, she was started on valproate sodium $250 \mathrm{mg}$ BID IV and an additional $250 \mathrm{mg}$ PRN for continued agitation, from days 10 to 13 . By day 14, the patient's RASS scores improved to 0 , and she was successfully extubated. Two scheduled doses of Valproate Sodium IV were administered to the patient on day 15 and then discontinued, as RASS scores remained at 0.

Lorazepam was discontinued due to the risk of respiratory depression, from oversedation with benzodiazepine therapy, in the setting of recovering COVID pneumonia. She was discharged on her home dose regimen of lithium and paliperidone for bipolar disorder.

\subsection{Case 2}

Case 2 concerns a 42-year-old female, with a history of bipolar disorder and post-traumatic stress disorder, who was similarly admitted for COVID pneumonia and acute hypoxic respiratory failure, requiring intubation. Her home regimen included quetiapine $400 \mathrm{mg}$ and alprazolam $2 \mathrm{mg}$ BID. The primary team consulted psychiatry for agitation in the setting of attempted extubation and delirium.

Initial management included holding home quetiapine, given the patient's acute hypoxia, her waxing and waning mental status, and the drug's known effect of causing further sedation and possible respiratory depression. The provider team also started the patient on haloperidol $2 \mathrm{mg}$ IV PRN for agitation on days 1 and 2. RASS scores were tracked during this time and ranged from -4 to +2 . By day 3, the patient's upper limit RASS score had increased to +3 , she was given lorazepam PRN for benzodiazepine withdrawal per Clinical Institute Withdrawal Assessment-Benzodiazepines (CIWA-B) (8 mg total PRN doses), and haloperidol $4 \mathrm{mg}$ IV therapy was initiated. Because of haloperidol's risk of prolonging the QTc interval, EKG monitoring was ordered, and the report subsequently showed a prolonged QTc interval (507 ms). Therefore, haloperidol was discontinued 
on day 4, to avoid further QTc prolongation and possible torsades arrhythmia in the patient. On day 5, valproate sodium IV $250 \mathrm{mg}$ BID scheduled dosing and valproate sodium IV $250 \mathrm{mg}$ PRN therapy was started, as a safer alternative to help manage patient agitation, without the risk of cardiotoxicity. During this time period, her RASS scores ranged from +2 to -1 and subsequently normalized to 0 . She was successfully extubated, with continued valproate sodium dosing. Valproate sodium IV was switched to oral formulation for 4 days, before being discontinued.

Patient 2 resumed her antipsychotic therapy with quetiapine on day 11 and subsequently developed fever (100.4 F) and tachycardia and demonstrated drooling and shuffling gait on exam. While extrapyramidal effects of quetiapine were strongly considered as a likely etiology, the psychiatry team was also concerned for catatonia, given the acuity and because this phenomenon can also cause autonomic derangements, including fever and tachycardia. Antipsychotic therapy was immediately discontinued, to reduce the risk of worsening symptoms of EPS versus febrile catatonia. The patient was restarted on valproic acid $250 \mathrm{mg}$ PO BID, and lorazepam $1 \mathrm{mg}$ TID and benztropine $1 \mathrm{mg}$ TID were also initiated as part of therapy. Clinical symptoms improved by day 17 , and the acute response to lorazepam and resolution of her autonomic signs, shuffling gait, and drooling supported febrile catatonia as the likely etiology. Valproic acid therapy was continued, and lorazepam was titrated downward. Upon discharge, the patient resumed home regimens of alprazolam and continued valproic acid, a switch from previous quetiapine, to help manage bipolar disorder.

\subsection{Case 3}

Case 3 describes a 23-year-old female, with a history of bipolar disorder with psychotic features and polysubstance abuse. She presented to our facility from an outside hospital, following cardiac arrest from illicit drug overdose. Home regimens included a paliperidone long acting injectable (LAI) every 3 months, and she was hospitalized just prior to her next scheduled dose.

Early during her hospital course, she developed acute kidney injury and acute limb ischemia, likely secondary to hypoperfusion from cardiac arrest. Unfortunately, the severity of limb ischemia required left lower extremity amputation, necessitating transfer to our hospital, to provide further resources pertinent to the procedure. Additionally, she also tested positive for COVID-19 and subsequently developed acute hypoxic respiratory failure, requiring intubation. The primary team consulted psychiatry for management of agitation in the setting of unsuccessful extubation attempts. RASS scores were trended and ranged from -3 to +2 during attempts. Initial management included oral valproic acid $250 \mathrm{mg}$ BID and $250 \mathrm{mg}$ PRN. Although IV valproate sodium therapy was the preferred formulation, due to its benefit of rapidity of action, a supply shortage required improvisation with the oral formulation. During her admission at our hospital, our team attempted to rectify home medication doses for her bipolar disorder, including her antipsychotic regimen. Because the patient was unable to confidently provide us her home regimen and dose, and no details were noted in the electronic medical record, our team contacted the outpatient community clinic, where she received treatment. The clinical staff were able to verify her paliperidone regimen and due dates. On day 11, the patient's valproic acid regimen was increased to $250 \mathrm{mg}$ at AM, 500 $\mathrm{mg}$ at PM, and $250 \mathrm{mg}$ BID PRN. This same day, the patient self-extubated, while RASS scores had stabilized to 0 on scheduled valproate sodium IV therapy. Valproate sodium was maintained on an adjusted regimen to assist with any recurrent signs of agitation, despite improved RASS scores. The 
patient was then started on risperidone $1 \mathrm{mg}$ BID for management of her bipolar disorder, as she was unable to receive her paliperidone LAI during her hospitalization. On day 16, valproate sodium was discontinued, because the patient's labs noted thrombocytopenia and decreased hemoglobin, causing concern for possible surgical site bleeding.

Following discontinuation of valproate sodium, the patient began displaying fever and tachycardia, as well as cogwheel rigidity of the upper extremities. These symptoms and autonomic signs raised concern for febrile catatonia. To reduce the risk of worsening catatonia, risperidone therapy was discontinued. The patient was then started on a lorazepam $0.5 \mathrm{mg}$ STAT trial, followed by a scheduled dosing of $1 \mathrm{mg}$ PO twice daily and $1 \mathrm{mg}$ at bedtime for catatonia. While higher titrated doses can further potentiate resolving catatonia symptoms, we maintained the patient at low doses of lorazepam, given her resolving COVID hypoxia and to reduce the risk of respiratory depression. The patient's catatonia symptoms appeared to partially respond to lorazepam; rigidity and fever resolved. However, tachycardia persisted. Several management modifications were made, including starting gabapentin and duloxetine. The rationale for adding this treatment was due to the patient's reported postoperative neuropathic pain, following amputation, and the reported therapeutic benefits of gabapentin and duloxetine. Gabapentin $900 \mathrm{mg}$ TID (eventually titrated downward to $600 \mathrm{mg}$ BID) PO and duloxetine $60 \mathrm{mg}$ PO daily were thus added, and the patient's vital monitoring noted resolution of her tachycardia. The patient was then discharged on quetiapine for continued management of her bipolar disorder, before her next scheduled LAI dosing.

\section{Discussion of Significance}

In all three cases, psychiatry was consulted for management of delirium and agitation in the setting of extubation. Typically, management in these conditions includes antipsychotics. In Case 1 , valproic acid was restarted, following delivery and with resolved concerns among providers for teratogenicity, along with antipsychotics. In Case 2, the patient was switched to valproate sodium after haloperidol was discontinued due to prolonged QTc intervals. In Case 3, valproic acid was given for agitation because of unclear information prior to extubation about the timing and type of the patient's home LAl antipsychotic. In all cases, valproate successfully managed agitation and delirium, as well as aided successful extubation.

In Cases 2 and 3, antipsychotic rechallenge was started after extubation for continued home regimen and standard therapy treatment of delirium and bipolar disorder. Subsequently, both cases developed catatonia, and antipsychotic medications were discontinued. In Case 2, valproate sodium was restarted. Valproate sodium could not be restarted in Case 3 due to surgical site bleeding. Catatonia was successfully managed and resolved in both cases, using lorazepam in Case 2 and lorazepam, diazepam, and gabapentin in Case 3.

The catatonia that resulted from antipsychotic rechallenge, along with the success of valproate sodium during agitation and delirium management in the setting of extubation, supports valproate sodium as an appropriate choice for these patients in managing delirium and catatonia. Additionally, the catatonia that resulted from antipsychotic rechallenge means further research on psychiatric management standards of COVID positive patients may be needed. At the time of writing, there have been few reported cases of catatonia in COVID patients [8, 9]. However, it has also been suggested that catatonia has been underdiagnosed in COVID patients due to limited physical exams, limited psychiatric evaluation, and the severity with which these patients present in general [10]. 
Cases 2 and 3 had clear signs of catatonia (e.g., tachycardia, fever, staring, cogwheel rigidity) and responded to standard catatonia treatments (e.g., lorazepam) and broad management (e.g., addition of gabapentin in Case 3). In Case 3, gabapentin and duloxetine were added due to their effects on neuropathic pain for the patient's post-amputation pain. An increased gabapentin dose was associated with the patient's improvement in pain symptoms, likely responsible for the resolution of tachycardia that has previously persisted despite improvement in the catatonia symptoms. Gabapentin has previously been reported as a treatment for catatonia and is proposed to improve catatonia via its glutamate antagonist properties [12, 13]. Further research into gabapentin's effects in aiding treatment of COVID catatonia may be warranted.

Moreover, the possibility that the patients in these cases had signs of catatonia, in addition to their diagnosed delirium, as they approached time of extubation must be considered. It is possible that a diagnosis of catatonia in medically complicated, isolated patients with COVID-19 respiratory failure could be missed. As antipsychotics are typically used for delirium and extubation-related agitation, it is possible that giving antipsychotics alone during this time would have worsened catatonia in an already complicated scenario of extubating a COVID-19 patient. It is worth considering that valproate use in these cases may have managed or prevented catatonia during extubation, in addition to managing delirium and agitation. In Cases 2 and 3, date of antipsychotic rechallenge closely followed date of valproic acid discontinuation. This opens the possibility that catatonia actually re-emerged as antipsychotics were restarted and valproate was no longer suppressing catatonia. The use of valproic acid in successful management of catatonia has been previously reported in case studies [14, 15].

As previously mentioned, the neuroinflammatory response of the CNS to COVID-19 infection, in particular the neutrophilic and monocytic infiltration that leads to disruption of the blood-brain barrier, may explain the poor neurological outcomes and delirium seen in COVID patients [7]. All three cases presented had evidence of oxidative stress caused by the SARS-CoV-2 virus peripherally (e.g., acute hypoxic respiratory failure) and centrally (e.g., agitation as a result of encephalopathy). Additionally, previous literature has shown that dexamethasone (used in Case 3) can cause neuropsychiatric complications after extubation due to its sedative and neuroinflammatory effects [6]. Data from a retrospective cohort study suggests that valproate sodium has been associated with reduced agitation and delirium among critically ill adults in the intensive care unit [16]. Several case series also reported improvement in ICU agitation or delirium with the use of valproate sodium [17, $18,19]$. Valproate has been suggested as an option for the managing delirium in critically ill patients due to its proposed biochemical mechanism, which provides anti-inflammatory and antioxidant effects [16, 20]. Valproic acid, a well-established and versatile antiepileptic drug, is known to have anti-inflammatory and antioxidative effects, and has been characterized as a neuroprotective drug based on evidence that it is beneficial in reducing neuronal death, balancing abnormal brain excitability, mitigating neurodegenerative events such as oxidative stress and protein oxidation induced by excitotoxicity, and regulating gene transcription through inhibition of histone deacetylases (HDACs) induced by ischemia, neuroinflammation, neurological deficits. [19, 22]. A case series on treatment of delirium and agitation in Bickerstaff encephalitis, which did not respond to standard management of neuroleptic agents but improved with valproic acid, also posits valproic acid's anti-inflammatory properties as a possible reason for improvement of symptoms, noting previous reports of its utility for delirium and agitation, including in traumatic brain injury [19].Valproate sodium therapy also provides the benefit of intravenous administration, making it a 
good candidate for management in the setting of extubation [21]. Given valproate's proposed mechanism, flexibility in administration route, and shown efficacy in the literature, we believe this case series complements current literature by showing valproate efficacy in management of agitation and delirium in COVID-19 patients. While not proposing that valproate is the new treatment standard for scenarios such as these, this case series highlights the usefulness of valproate in patients with COVID-19 who present with agitation during extubation, delirium that is difficult to manage in the context of COVID-19 related medication contraindications, and when catatonia and delirium are difficult to differentiate.

\section{Author Contributions}

Schumaker A, Gomez R, Rojasova S, Muir C, Meresh E., drafted the manuscript, contributed in manuscript writing, reviewed literature, revised and edited the manuscript, Bukiri K, Korneffel $C$, Sharma S, Rao M., reviewed literature, provided feedback, revised and edited the manuscript.

\section{Funding}

This research did not receive any specific grant from funding agencies in the public, commercial, or not-for-profit sectors.

\section{Competing Interests}

The authors report no proprietary or commercial interest in any product mentioned or concept discussed in this article.

\section{References}

1. Beach SR, Praschan NC, Hogan C, Dotson S, Merideth F, Kontos N, et al. Delirium in COVID-19: A case series and exploration of potential mechanisms for central nervous system involvement. Gen Hosp Psychiatry. 2020; 65: 47-53.

2. Varatharaj A, Thomas N, Ellul MA, Davies NW, Pollak TA, Tenorio EL, et al. Neurological and neuropsychiatric complications of COVID-19 in 153 patients: A UK-wide surveillance study. Lancet Psychiatry. 2020; 7: 875-882.

3. Vajda FJ. Valproate and neuroprotection. J Clin Neurosci. 2020; 9: 508-514.

4. Amouri J, Andrews PS, Heckers S, Ely EW, Wilson JE. A case of concurrent delirium and catatonia in a woman with coronavirus disease 2019. J Acad Consult Liaison Psychiatry. 2021; 62: 109114.

5. Tango RC. Psychiatric side effects of medications prescribed in internal medicine. Dialogues Clin Neurosci. 2003; 5: 155-165.

6. Warrington TP, Bostwick JM. Psychiatric adverse effects of corticosteroids. Mayo Clin Proc. 2006; 81: 1361-1367.

7. Baller EB, Hogan CS, Fusunyan MA, Ivkovic A, Luccarelli JW, Madva E, et al. Neurocovid: Pharmacological recommendations for delirium associated with COVID-19. Psychosomatics. 2020; 61: 585-596.

8. Caan MP, Lim CT, Howard M. A case of catatonia in a man with COVID-19. Psychosomatics. 2020; 61: 556-560. 
9. Gouse BM, Spears WE, Archibald AN, Montalvo C. Catatonia in a hospitalized patient with COVID-19 and proposed immune-mediated mechanism. Brain Behav Immun. 2020; 89: 529-530.

10. Cooper JJ, Ross DA. COVID-19 catatonia-would we even know? Biol Psychiatry. 2020; 88: e19e21.

11. Wilson JE, Carlson R, Duggan MC, Pandharipande P, Girard TD, Wang L, et al. Delirium and catatonia in critically ill patients: The delirium and catatonia prospective cohort investigation. Crit Care Med. 2018; 45: 1837-1844.

12. Neuhut R, Levy R, Kondracke A. Resolution of catatonia after treatment with stimulant medication in a patient with bipolar disorder. Psychosomatics. 2012; 53: 482-484.

13. Rosebush PI, MacQueen GM, Mazurek MF. Catatonia following gabapentin withdrawal. J Clin Psychopharmacol. 1999; 19: 188-189.

14. KrÜger S, BrÄunig P. Intravenous valproic acid in the treatment of severe catatonia. J Neuropsychiatry Clin Neurosci. 2001; 13: 303-304.

15. Bowers R, Ajit SS. Is there a role for valproic acid in the treatment of catatonia? J Neuropsychiatry Clin Neurosci. 2007; 19: 197-198.

16. Gagnon DJ, Fontaine GV, Smith KE, Riker RR, Miller III RR, Lerwick PA, et al. Valproate for agitation in critically ill patients: A retrospective study. J Crit Care. 2017; 37: 119-125.

17. Bourgeois JA, Koike AK, Simmons JE, Telles S, Eggleston C. Adjunctive valproic acid for delirium and/or agitation on a consultation-liaison service: a report of six cases. J Neuropsychiatry Clin Neurosci. 2005; 17: 232-238.

18. Sher Y, Miller AC, Lolak S, Ament A, Maldonado JR. Adjunctive valproic acid in managementrefractory hyperactive delirium: A case series and rationale. J Neuropsychiatry Clin Neurosci. 2015; 27: 365-370.

19. Alam A, Puri NV. Inefficacy of antipsychotics in treatment of delirium and agitation in two cases of bickerstaff brainstem encephalitis. J Neuropsychiatry Clin Neurosci. 2014; 26: 176-178.

20. Sher Y, Cramer AC, Ament A, Lolak S, Maldonado JR. Valproic acid for treatment of hyperactive or mixed delirium: Rationale and literature review. Psychosomatics. 2015; 56: 615-625.

21. Norton JW, Quarles E. Intravenous valproate in neuropsychiatry. Pharmacotherapy. 2000; 20: 88-92.

22. Machado, Ximenes JC, Lima, Verde CE, Naffah-Mazzacoratti MdG, et al. Valproic acid, a drug with multiple molecular targets related to its potential neuroprotective action. Neurosci Med 2012; 3:107-123 


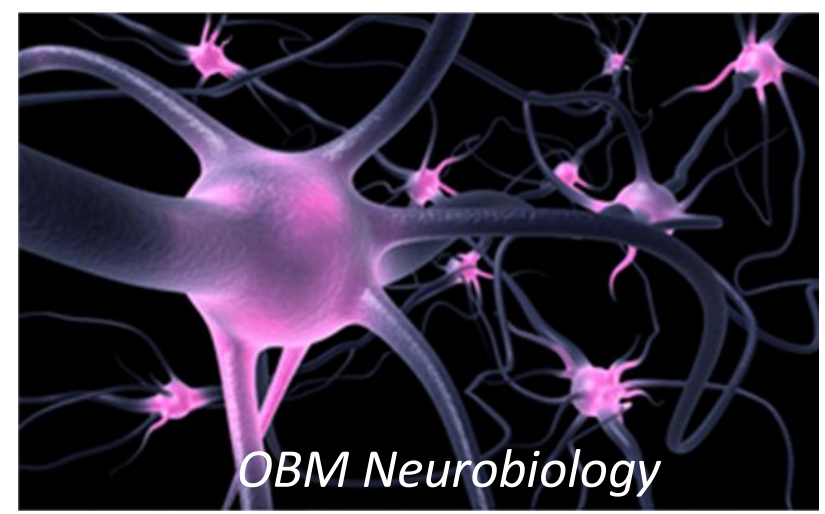

Enjoy OBM Neurobiology by:

1. Submitting a manuscript

2. Joining volunteer reviewer bank

3. Joining Editorial Board

4. Guest editing a special issue

For more details, please visit:

http://www.lidsen.com/journals/neurobiology 